\title{
An Intelligent Home-based Monitoring System for the Elderly
}

\author{
Wei Wu \\ Chongqing City Management College \\ Chongqing 401331 \\ China \\ wwzj_cq@qq.com
}

\begin{abstract}
The existing monitoring system for nursing homes is not suitable for deploying in the common residential buildings and it require the manager to proactively find whether the elderly are in danger. An new intelligent monitoring system was proposed in this paper, which was aiming at the new needs of home-based care for the aged that live in the common residential buildings and need to call for help automatically when they encounter the unexpected accident. In the intelligent monitoring system, the UWB wireless positioning technology is used to monitor the current location of the elderly and a LoRa-based data-concentrator is utilized to obtain the information from the card readers carried by the old people and send the information to the management server of the system. According to the received information from the data-concentrator, once an emergency happens to the monitored old people, the monitoring system triggers the emergency response mechanism to send rescue information to managers of the system automatically, so that the rescue was carried out in time. Finally experimental results have shown the novel monitoring system can effectively monitor the safety of the elderly living alone and ensured their life security.
\end{abstract}

\section{CCS CONCEPTS}

- Computer systems organization $\rightarrow$ Embedded systems;

\section{KEYWORDS}

Home-based endowment, intelligent monitoring, UWB, LoRa, label

\section{INTRODUCTION}

Nowadays increasing old people in our country will require more elder care. Therefore, it has become an urgent and serious topic of our country to properly solve the demand of a large population's old-age life. However, there are not enough public

Permission to make digital or hard copies of all or part of this work for personal or classroom use is granted without fee provided that copies are not made or distributed for profit or commercial advantage and that copies bear this notice and the full citation on the first page. To copy otherwise, to republish, to post on servers or to redistribute to lists, requires prior specific permission and/or a fee.

MOBIMEDIA 2017, July 13-14, Chongqing, People's Republic of China Copyright @ 2017 EAI 978-1-63190-156-0

\author{
Lijun Tang \\ Chongqing Vocational Institute of \\ Engineering \\ Chongqing 402660 \\ China \\ tanglijun_2002@163.com
}

elderly are mostly general residential housing, the existing monitoring system for nursing homes is no longer adapt to the new mode of home-based endowment. Therefore, those elderly people that live alone in their home can't get good care. When they encountered the specific emergencies, the existing monitoring system can not automatically send the alarm to the others to help them, so that the elderly will be in danger. Thus the old monitoring systems need to be improved to adapt the new endowment mode.

In this paper, a wireless intelligent monitoring system is designed for the home-based endowment mode, utilizing UWB (Ultra Wide Band) technology which can correctly find the location of the elderly who live in home alone and monitor their daily life in case of some emergencies. It can implement the real-time positioning, so that the safety monitoring of indoor activities and rescuing problem for the elderly have been solved effectively [1-3].

Furthermore, the proposed monitoring system combines with efficient computer network to notify the community managers of the emergency rescue on the elderly. It can fully provide many convenient services for the elderly. Therefore, it could be used to the home-based endowment situation widely in the near future.

\section{Design of intelligent monitoring system}

\subsection{Structure of intelligent monitoring system}

Generally, the intelligent monitoring system is mainly composed of a management server, a data-concentrator, several card readers and personnel labels [4-7]. The functions of the management server are storing and managing data, printing management report, sending service information of the elderly to their children's mobile phones and so on. The data-concentrator is responsible for collecting the information from the card reader based on the LoRa(Long Range) technology. And the card readers collect the information from labels. These labels are wore by the elderly, and it can send the positioning data and help information about the aged people to the card reader.

For the home-based endowment, the elderly always lives in the ordinary residential buildings where there is no existing special network to serve for the home-based monitoring system. In order to facilitate the deployment, reduce the wiring, control the cost of the equipment and improve the system extensibility, we adopts LoRa to transmit and manage data in the system. LoRa is a kind of long range and low power consumption wireless transmission technology, so it is fit for the home-base monitoring system. 


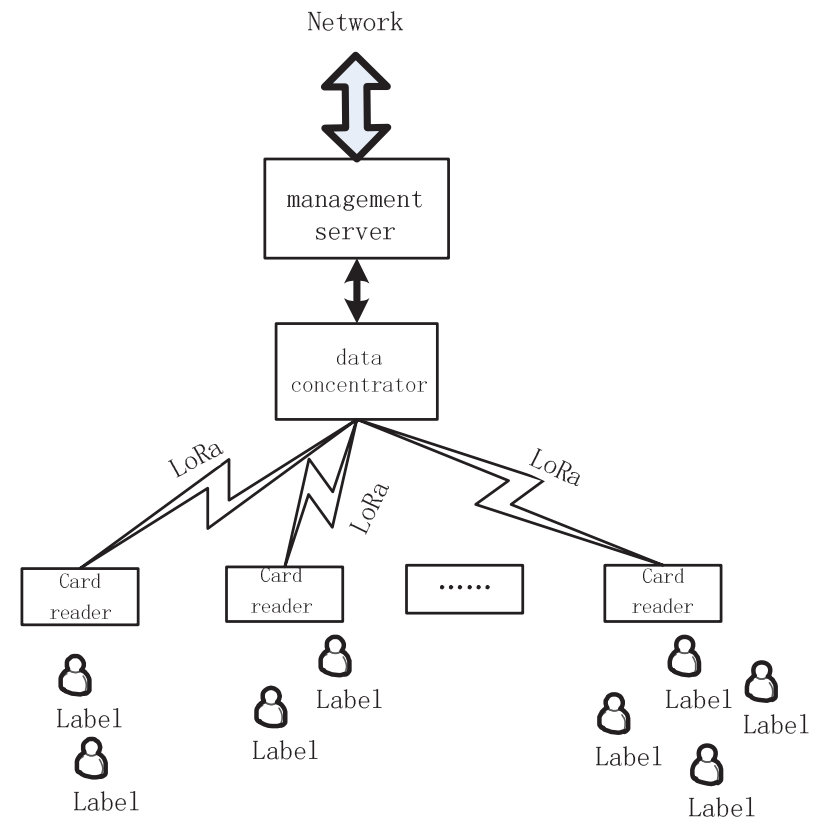

Figure 1: Topology of Intelligent monitoring system

Figure 1 shows the topology of the intelligent monitoring system for home-based endowment. In this topology, each old people in the rooms wears a label, the labels are responsible for collecting information from the old man that wears it, then transmitting these information to the card readers which are deployed in each room of the residential, and the card readers send the information from labels to the data-concentrator that is laid in the special room of the residential building. The data-concentrator utilizes the LoRa to receive the information from the labels that the old men wear and uploads the information to the management server. According to the data uploaded by the management server, the identity information, real-time position information and time information of the wearer are determined. At the same time, the information can been sent to the mobile phone through the public network, so the elderly family can know the real-time state of the elderly.

In practical application, the intelligent monitoring system that can meet the requirements of the modern endowment community has its particularities. First of all, the intelligent monitoring system is mainly used for indoor positioning, therefore it's positioning accuracy cannot exceed a few meters. Secondly, when an emergency occurs, the monitoring system can receive the alarm information timely and send it to the managers within a few seconds. Thirdly, due to the elderly living apart in residential buildings, the system should have the flexible networking capability. According to the above requirements, the intelligent monitoring system needs some special technologies to meet these practical requirements of the home-based endowment, which include the accurate positioning technology, wireless transmitting technology (LoRa), hardware and software, and the efficient emergency response mechanism

\subsection{Accurate positioning technology}

The intelligent monitoring system is based on TOA (Time of arrive) [8], it is a positioning algorithm of UWB technology and it uses the two-way distance measurement method to calculate the distance between two nodes. And the TOA can improve the positioning accuracy of the system, and it can be used to adjust the power consumption of the label that is worn by the elderly.

In the monitoring system, the card reader using TOA positioning algorithm can accurately calculate the distance from it to the personnel labels and send the distance to the management server periodically, and the positioning accuracy error is less than 1 meter. According to the distance provided by the card reader, the management server can accurately infer where the elderly is.

\subsection{LoRa technology}

In the intelligent monitoring system, LoRa, a kind of wireless communication technology, is utilized to serve for the endowment community. The transmission distance that LoRa can achieve is up to 2 kilometers, and the communication using LoRa can not be hampered by the buildings, so it is possible to achieve remote data transmission by LoRa.

In addition, LoRa make the deployment of the our monitoring system more flexible and convenient than the existing monitoring system for the nursing home. With LoRa, the card readers can be installed in any room of the residential buildings without wired connection. In the control room, it is equipped with the dataconcentrator that can gather the information from all card readers through the wireless network and send the information to the management server.

\subsection{Emergency response mechanism}

In the proposed monitoring system, once an emergency on the elderly occurs, we would utilize an efficient emergency response mechanism to rescue the elderly.

At first we observed the regular life of the elderly in doors for several weeks and found the law of their living, then set an timethreshold for each room of their home according to the time table of their daily life. The time-threshold is the longest time that the elderly usually keep still in the room in a particular duration .

If the elderly encountered the special emergencies, like falling, coma or other serious illness, in such situation they weren't able to press the help button, even they could not move. When the management server find the old people keep still in the same location according to the received periodic information, and the still time exceeded the setting time-threshold in advance, the management server would judge that some emergencies happened to the elderly, and it automatically notify managers to rescue the elderly. For example, if an old people should get up at eight o'clock in the morning usually, but one day he or she still stayed on the bed at eight o'clock and maintained the time beyond the time-threshold which was set in advance, we would infer it was more likely that the old people encountered some emergencies, such as falling, coma.

The processing procedures of the emergency response mechanism is shown in Figure 2. When an elderly falls in the room, the alarm immediately is triggered automatically, then the management 
server will notify the managers about the emergency, and the manager will provide the emergency rescue soon. When an elderly manually triggers the help button on the label, the management server informs the managers that someone needs help. Or the time that the elderly keep still in the same location exceeds the threshold time setting on the management server; the management server also will notify the managers pay attention to the unmovable elderly.

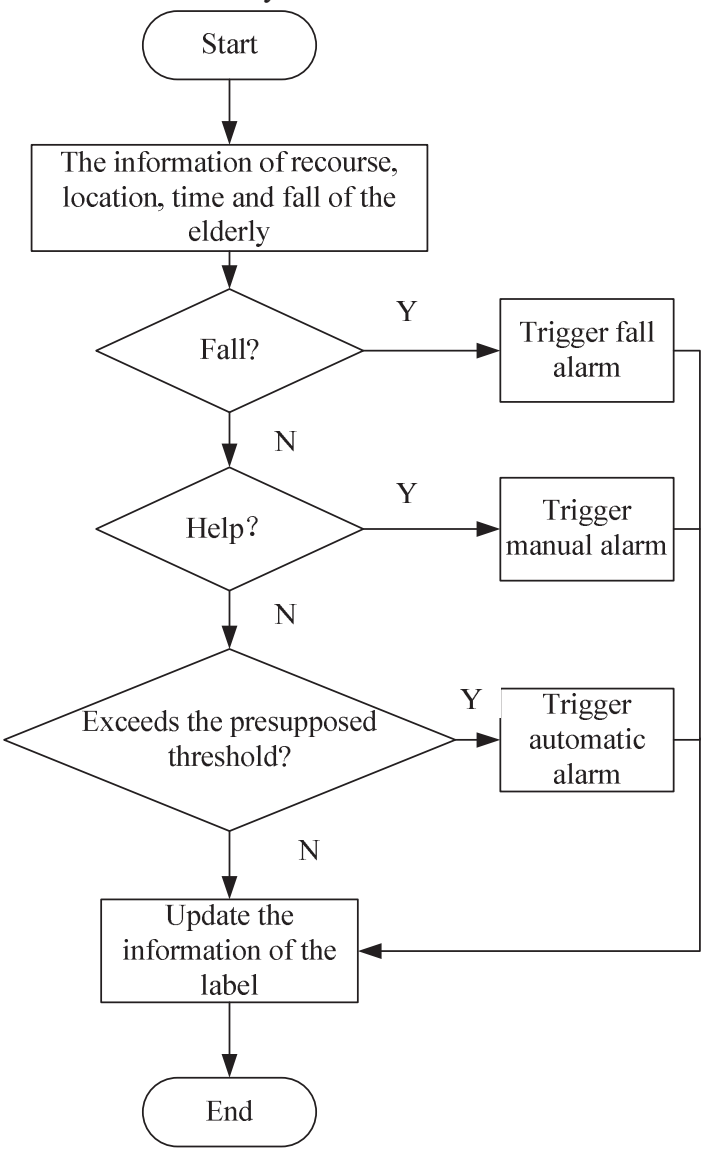

Figure 2: Emergency response mechanism

\subsection{Hardware for intelligent monitoring system}

The main hardware for the intelligent monitoring system includes the personnel labels and the card readers. The personnel label is composed of the UWB positioning module, the micro controller, the fall detection module and the power management module. The card reader is composed of the UWB positioning module, the LoRa wireless communication module, the power management module and the micro controller.

The UWB positioning module is used to obtain the distance between the card reader and the label. The LoRa wireless communication module is used to send the data to the dataconcentrator by the card reader. The power management module is used to save the overall battery power of the system. When the label moves out of the range of the card reader, the power management module keeps the car reader be asleep and save its power.

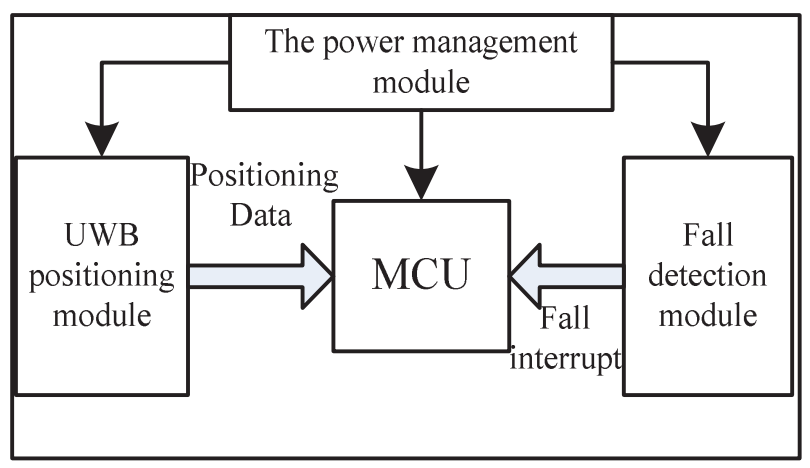

Figure 3: Principle diagram of the label

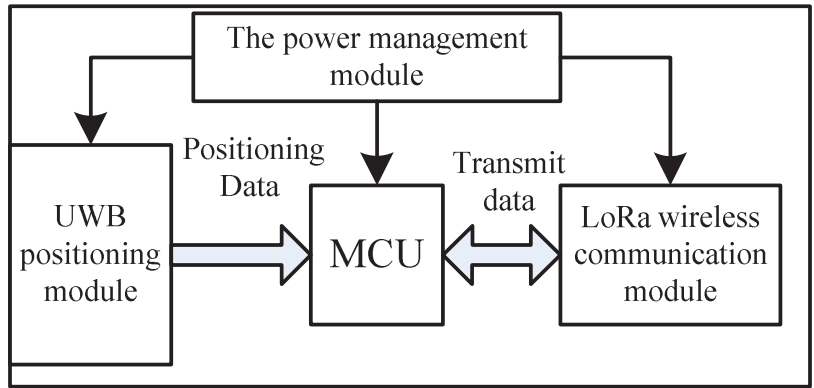

Figure 4: Principle diagram of the card reader

\subsection{Software for intelligent monitoring system}

In this system, the software for the management server has many kinds of basic functions and expanding functions, which are suitable for the home-based endowment $[9,10]$.

The software for management server can show the "elderly position" in real time. And it can provide the track inquiry, the historical record inquiry. According to the special group of the elderly and the reasonable management mechanism, the software can optimize the rescue alarm mechanism and the management mechanism of the elderly. The functions of the management software are divided into 5 parts as follows:

Map display --- Displays the elderly location in real-time on the map.

Information inquiry --- Provides the historical records of the location and alarm of the elderly.

Management of base station --- Manages the setting of the card readers.

Personnel management --- Manages the personal information of the managers and the elderly, including query, edit, add, delete and other operations.

System functions --- Including the modification of the login password, log out and other functions. 


\begin{tabular}{|c|c|c|c|c|}
\hline \multicolumn{5}{|c|}{ WEBPAGE } \\
\hline $\begin{array}{c}\text { Map } \\
\text { display }\end{array}$ & $\begin{array}{c}\text { Information } \\
\text { inquiry }\end{array}$ & \begin{tabular}{|c|} 
Management \\
of base \\
station
\end{tabular} & $\begin{array}{c}\text { Personnel } \\
\text { management }\end{array}$ & $\begin{array}{c}\text { System } \\
\text { functions }\end{array}$ \\
\hline
\end{tabular}

Figure 5: Software block diagram for management server

\section{Results of intelligent monitoring system}

The intelligent monitoring system is mostly built in the general residential building; its construction is shown in Figure 1.

The card readers are installed in one room of the residential building, and the data-concentrator and the management server are placed in other residential building nearby.Assumed that that the elderly usually do not stay still in the bedroom for more than 15 minutes during the day,so the time-thresholds is set to 15 minutes.

Let a tester carried a label walk in doors, then the tester keep still in the bedroom for more than 15 minutes. We simulated the fall, coma, serious illness and other emergency situations in the experiment, the alarm rate of different emergencies are shown in table 1.

Table 1: Alarm rate of emergency response mechanism

\begin{tabular}{lcc}
\hline Parameter & Test contents & Alarm Rate \\
\hline Manual alarm & $\begin{array}{c}\text { Triggers the help button } \\
\text { Fall rescue }\end{array}$ & $\begin{array}{c}\text { Simulates the falls of the } \\
\text { elderly }\end{array}$ \\
$\begin{array}{c}\text { Emergency } \\
\text { notification }\end{array}$ & $\begin{array}{c}\text { Simulated emergencies } \\
\text { of the elderly }\end{array}$ & $\geqslant 97.5 \%$ \\
\hline
\end{tabular}

When the elderly triggered the help button on the label, the label will upload the manual alarm to the manager server. And the upload rate of the manual alarm was up to $100 \%$. Once the elderly falls, the label can detect the violent change of the acceleration, and then the label uploads the fall rescue to the manager server immediately. And the alarm rate of the fall rescue is greater than 97.5\%.When the coma, serious illness and other emergency situations are occurred, the elderly will remain still for an abnormal long time. Under these circumstances, the manager server can judge the elderly need help, so the system generates an emergency notification. And the alarm rate of the emergency notification is greater than $95.5 \%$. When the alarm is timely received by the manger, the elderly will be rescued soon. So these experimental results showed the response mechanism can find the unexpected accident on the tester accurately .

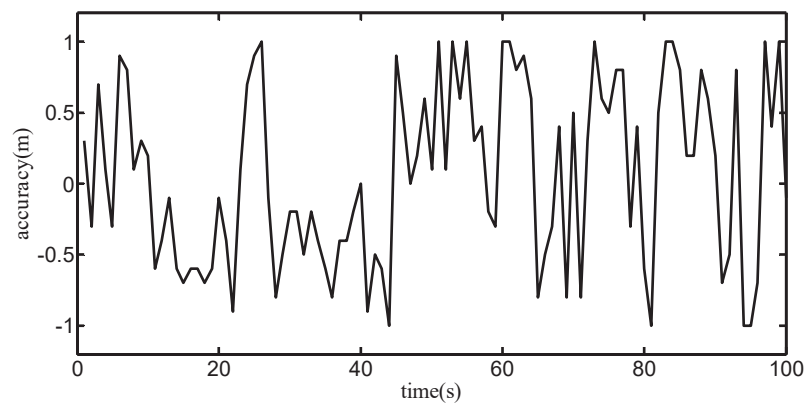

Figure 6: Accuracy of the intelligent monitoring system

The accuracy of the intelligent monitoring system is plotted in Fig. 6. It displays that the accuracy of the system is less than one meter. So the system can use the position data to accurately judge the elderly is still or not.

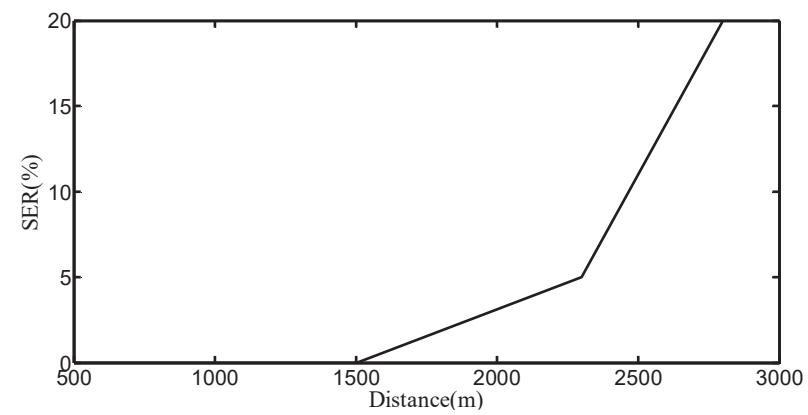

Figure 7: Transmission distance of the intelligent monitoring system

Fig.7 illustrates the transmission distance of the intelligent monitoring system. The intelligent monitoring system can achieve 1500 meters of wireless signal transmission without SER (symbol error rate) by using LoRa technology. And the SER of the wireless signal transmission at 2000 meters is less than 5\%. Therefore, the intelligent monitoring system can be used to replace the existing wired signal transmission between the card reader and the management server by using LoRa technology. It is greatly simplifying the intelligent monitoring system deployment costs and difficulties.

\section{CONCLUSIONS}

The existing intelligent monitoring systems for nursing homes cannot adapt to the new home-based endowment any longer. To resolve the above problem, the wireless positioning technology: UWB and the wireless transmission technology: LoRa are used in our proposed monitoring system. Thus the monitoring system can be deployed more easily than the old one, it's cost is relatively low, and it can meet the demand of the home-based endowment. Through a large amount of experiments, the monitoring system can provide safety protection for the elderly living in home; 
therefore it would have a big application prospect in home-based endowment market.

\section{A HEADINGS IN APPENDICES}

\section{A.1 Introduction}

\section{A.2 Design of intelligent monitoring system}

\section{A.2.1 Structure of intelligent monitoring system}

A.2.2 Accurate positioning technology

A.2.3 LoRa technology

A.2.4 Emergency response mechanism

A.2.5 Hardware for intelligent monitoring system

A.2.6 Software for intelligent monitoring system

\section{A.3 Results of intelligent monitoring system}

A.4 Conclusions

\section{A.5 References}

\section{ACKNOWLEDGMENTS}

This work was supported by the Science and Tech. Research Fund Project of Chongqing Education Commission (KJ1603107).

\section{REFERENCES}

[1] Xiao Tiejun, Chen Xiao.Wireless positioning system in the application research of endowment communities[J]. Electrotechnical Application, 2014,(7):86-90.

[2] Liu Shulun, Wang Shusen.Underground personnel positioning system based on ultra wideband technology[J].Indstry and mine automation,2014,40(10):81-83.

[3] Xiao Zhu,,Wang YongChao, Tian Bin,etc. Development and prospect of UltraWideband localization research and application[J].Acta Electronica Sinica,2011,39(1):133-141.

[4] Zhong Min,Tang KaiYe,Qiu Liang.Design and implementation of endowment and old aiding interaction system based on wireless sensor network[J].Modern Architecture Electric,2013,4(4):9-14.

[5] Wang Tuo.Elderly intelligent community cloud storage and cloud services research[D].University of Chinese Academy of Sciences, 2013.

[6] Qiu Ming.The intelligent community home endowment system based on ZigBee technology of the Internet of things[J].Journal of Foshan University(Natural Science Edition), 2015,(2):71-75.

[7] Du XinFeng,Qin Yan.Design of home-based medical care system for aged based on wireless network [J].Chinese Medical Equipment Journal,2014, 35(10):39-41.

[8] Wu Wei, Liu Shisen.Accurate person positioning system for coal mine based on TOA technique[J].China Coal,2012,38(4): 65-67.

[9] WANG Hong, BIAN Hong-ning, GONG Lin-lin.The Software Design of Intelligent Health Monitoring System[J].Value Engineering,2014, (32):257-259.

[10] Zhang ShiFu, Jiang Tao, Wu XiaoMing.Design and implementation of health

care management system software in intelligent community based on

$\mathrm{b} / \mathrm{s}[\mathrm{J}]$.Computer Applications and Software,2014,(3):4-6. 